\title{
Transgenic expression of DwMYB2 impairs iron transport from root to shoot in Arabidopsis thaliana
}

\author{
Yan-Hong Chen ${ }^{1,2,3}$, Xue-Min $\mathrm{Wu}^{5}$, Hong-Qing Ling ${ }^{4}$, Wei-Cai Yang ${ }^{1}$ \\ ${ }^{1}$ The Key Laboratory of Molecular and Developmental Biology, Institute of Genetics and Developmental Biology, Chinese Academy \\ of Sciences, Beijing 100101 China; ${ }^{2}$ Graduate University of the Chinese Academy of Sciences, 19 Yuquan Road, Beijing 100049 , \\ China; ${ }^{3}$ School of Life Science, Nantong University, Nantong 226007, China; ${ }^{4}$ The State Key Laboratory of Plant Cell and Chromo- \\ some Engineering, Institute of Genetics and Developmental Biology, Chinese Academy of Sciences, Beijing 100101, China
}

In plant, iron uptake and homeostasis are tightly regulated to ensure its absorption from soil and to avoid excess iron in the cell. Many genes involved in this process have been identified during past several years, but there are many problems remain unsolved in the genetic regulation of whole plant iron trafficking and allocation. MYB transcription factors contain tandem repeats of a $\sim 50$ amino acid DNA-binding motif (R) and are involved in the regulation of many aspects of plant development, hormone signaling and metabolism. Here, we report that the ectopic expression of orchid R2R3-MYB gene DwMYB2 in Arabidopsis thaliana confers the transgenic plants hypersensitivity to iron deficiency. In $D w M Y B 2$ transgenic plants, the iron content in root is two-fold higher compared to that in wild-type root, while the reverse is true in shoot. This imbalance of iron content in root and shoot suggested that the translocation of iron from root to shoot was affected by the expression of $D w M Y B 2$ in the transgenic plants. Consistently, gene chip and reverse transcription-polymerase chain reaction analysis revealed that the ferric-chelate reductase gene, AtFRO2, and the iron transporter gene, AtIRT1 and AtIRT2, are up-regulated by DwMYB2 expression, while other potential iron transporters such as AtIREG1, AtFRD3 and NRAMP1 are down-regulated. In addition, the expression of several putative peptide transporters and transcription factors are also altered in the $35 S:: D w M Y B 2$ transgenic lines. These data provide us insight into the whole plant translocation of iron and identify candidate genes for iron homeostasis in plants despite the fact that a heterologous gene was expressed.

Cell Research (2006) 16:830-840. doi:10.1038/sj.cr.7310099; published online 10 October 2006

Keywords: DwMYB2, iron uptake and homeostasis, iron transporters, Arabidopsis

\section{Introduction}

Iron is an essential element for all organisms including plants. It functions as electron acceptor and donor and thus participates in a number of important redox reactions for respiration and photosynthesis. Although iron is abundant in the Earth's crust, it is often unavailable to

\footnotetext{
${ }^{5}$ Current address: Department of Botany, University of British Columbia, 6270 University Blvd., Vancouver, British Columbia, Canada V6T 1 Z4.

Correspondence: Wei-Cai Yang

Tel: +86-010-6255-1272; Fax: +86-010-6255-1272;

E-mail: wcyang@genetics.ac.cn

Received 12 Jun 2006; revised 19 Jul 2006; accepted 29 Aug 2006; published online 10 Oct 2006
}

plants because it tends to form insoluble ferric hydroxide complex in aerobic environment [1]. In addition, Fe(II) and $\mathrm{Fe}$ (III) act catalytically to generate hydroxyl radicals that can damage cellular constituents such as DNA and lipids [2]. Therefore, iron uptake, storage and metabolism must be carefully regulated and iron homeostasis has to be maintained. The strategy I plants including dicots and nongrass monocots respond to iron deficiency by the release of protons, resulting in acidification of the rhizosphere, which drives more Fe(III) into solution. Then, Fe(III) at the root surface is reduced by ferric reductases to $\mathrm{Fe}(\mathrm{II})$, which is subsequently transported across the root epidermal cell membrane via iron transporters [3]. Finally, iron in roots has to be transported to the shoot, where it participates in photosynthesis and respiration. Several key genes partici- 
pating in iron uptake have been identified in Arabidopsis [4-7], tomato [8-11] and pea [12]; however, genes and mechanisms controlling the root-to-shoot transportation of iron remain to be revealed.

Under iron-deficient conditions, these plants acidify the soil through activation of a specific plasma membrane $\mathrm{H}^{+}$-ATPase of the root epidermal cells, potentially encoded by the AHA2 gene in Arabidopsis [13]. Consequently, iron solubility increases, and $\mathrm{Fe}(\mathrm{III})$ chelates are reduced by a specific root reductase such as ferric-chelate reductase AtFRO2 from Arabidopsis [6] and PsFRO1 from pea [12] as well as LeFRO1 from tomato [11]; the Fe(II) was then transported across the root plasma membrane via ferrous iron transporters AtIRT1 and AtIRT2 [5, 7, 14, 15]. AtIRT1, PSFRO1 and AtFRO2 as well as LeFRO1 play a key role in root iron uptake in response to iron deficiency. These genes are rapidly induced in response to iron starvation in roots $[5,6,11,12,15]$ and quickly turned off in iron sufficiency [16]. Furthermore, AtIRT1 and AtFRO2 are strictly co-regulated by local iron availability and a shoot-born systemic signal [17].

Because of its easy oxidization and limited solubility, Fe ions have to be chelated or stored in vacuoles after uptake into the cell. This implies that numerous iron chelators and transporters are involved in iron homeostasis. The main chelator in higher plants is possibly the non-proteinogenic amino acid nicotianamine (NA), a ubiquitous chelator for iron and other metals in plant [18]. Then long-distance iron transport between organs and tissues and intracellular iron transport for compartmentalization within organelles are required to complete numerous cellular functions and maintain iron homeostasis in plant cell, tissue and organs. Members of the Arabidopsis NRAMP transporter family have been shown to be involved in iron homeostasis [19, 20]; however, their physiological role in plants remains unclear. Plant NRAMP transporters could be involved in the compartmentalization of metals at the cellular level $[19,20]$. The multidrug and toxin efflux (MATE) family of transmembrane efflux protein, FRD3, is another iron transporter that is more likely to be involved in perception of the shoot-derived signal since $\mathrm{frd} 3$ mutant constitutively express AtIRT1 and AtFRO2 irrespective of the iron availability [21]. Other transporters such as iron-regulated gene1 (IREG1) [22], with a possible function in xylem loading, and yellow stripe-like proteins (YSLs) [23] are also reported, but the precise role of these transporters in iron allocation and homoeostasis in plant cells remains elusive.

The study of regulation mechanism of iron uptake is still in its infancy, although some progress has been made. The putative basic helix-loop-helix (bHLH) transcription factors, FER in tomato [10] and its ortholog FIT1 in Arabi- dopsis [4, 24], were potential regulators in iron deficiency responses. MYB transcription factors contain a $\mathrm{C}$-terminal domain and an $\mathrm{N}$-terminal conserved DNA-binding domain that is composed of tandem repeats of a $\sim 50$ amino acid helix-turn-helix motif. In plant, $M Y B$ genes are involved in the regulation of many aspects of plant development, hormone signaling and metabolism, such as epidermal cell differentiation, leaf development, signal transduction in salicylic acid, abscisic acid, gibberellic acid and jasmonic acid pathways, and phenylpropanoid biosynthesis [25, 26]. In microarray analysis of fit 1 mutant, the expression of two MYB genes was up-regulated by iron deficiency [4], suggesting that $M Y B$ genes perhaps also play a role in iron homeostasis. DwMYB2, initially isolated from orchid Dendrobium hybrid Woo Leng, represents a typical plant R2R3-MYB gene [27]. Here we report that DwMYB2 expression in transgenic Arabidopsis impaired iron translocation from root and shoot, resulting in iron accumulation in root and deficiency in shoot. DNA microarray and reverse transcription-polymerase chain reaction (RT-PCR) analysis showed that the expression of iron-responsive genes, such as AtIRT1, AtFRO2, AtFRD3, AtIREG1 and AtNRAMP1, were altered in transgenic roots and/or shoots. These data provide novel insights for the regulation of genes involved in iron transport and identified potential candidate genes for further investigation.

\section{Materials and Methods}

\section{Plant materials and transformation}

Arabidopsis thaliana ecotype Landsberg erecta was grown under $16 \mathrm{~h}$ light $/ 8 \mathrm{~h}$ dark cycle at $20-24{ }^{\circ} \mathrm{C}$ in a greenhouse. Arabidopsis seeds were surface sterilized subsequently with $10 \%$ bleach and $70 \%$ ethanol for $10 \mathrm{~min}$, rinsed five times with sterile water and finally plated on MS or B5 basal medium supplemented with $1 \%$ sucrose with $20 \mathrm{mg} \mathrm{ml}^{-1}$ hygromycin for $35 S:: D w M Y B 2$ transgenic plants or without the antibiotic selection for wild-type plants. Sterile seeds were kept at $4{ }^{\circ} \mathrm{C}$ for 2-3 days before transfer to growth chamber. $\mathrm{T} 2$ seeds were also directly sowed to potting soil and grew in green house. For iron treatment, MS medium was prepared without iron, and FeNaEDTA was supplied as liquid to the control MS plates. T4 seedlings of $35 S:: D w M Y B 2$ transgenic line DwMYB2-1 were used to do the following experiment. Arabidopsis was transformed via Agrobacterium-mediated vacuum infiltration method [28].

\section{Molecular cloning}

The 35S::GUS::NOS cassette was released from pBI121 (Clontech) with EcoRI and HindIII digestion, and subcloned to pCAMBIA1300 at EcoRI and HindIII site. The GUS gene in the cassette was then replaced by a full-length $D w M Y B 2$ cDNA clone (GenBank accession number: AF485893) [27] at $\mathrm{XbaI}$ and $\mathrm{BamHI}$ site to give rise to $p 35 S:: D w M Y B 2$.

\section{Northern blot analysis}

Total RNA was extracted from 40-day-old whole plant using Trizol 
reagent (Gibco), and RNA concentration and purity were determined by spectrometry. Five micrograms or less total RNAs were loaded on each lane and fractionated in $1.2 \%$ formaldehyde-containing agarose gel [42], then transferred onto Hybond-N membrane (Amersham) following the manufacturer's instructions and cross-linked to the membrane by a UV cross-linker (Bio-Rad). The membranes were hybridized with RNA probes, which were labeled with digoxigenin (Roche) and detected with the Chemiluminescent CDP-Star kit (Roche) in accordance with the manufacturer's instructions.

\section{Scanning electron microscopy}

Tissues and seedlings were frozen in liquid nitrogen, and the frozen samples were observed with JSM-5310LV scanning electron microscope (SEM) (JEOL Ltd, Japan). Pictures obtained were edited with Photoshop 7.0 software (Adobe).

\section{Real-time PCR and semi-quantitative PCR}

RNA was treated with DNase I to remove DNA contamination. First-strand cDNA was prepared by using Superscript RT (Invitrogen) and diluted 20-fold in water for RT-PCR. PCR was performed in a LightCycler (Roche) with the LightCycler-FastStart DNA Master SYBR Green I Kit (Roche) according to the manufacturer's protocols. cDNA quantities in each reaction were calculated by using LightCycler 3.1 software (Roche). All quantifications were normalized to $A C T I N$ cDNA fragments.

For semi-quantitative PCR, the first-strand cDNA was used as template for PCR amplification of cDNA fragment. PCR was performed for different cycles for different gene. The primers for real-time PCR and semi-quantitative PCR are summarized in Supplement 1.

\section{Assay of ferric-chelate reductase activity}

Wild-type (Ler) and 35S::DwMYB2 transgenic plants were grown for 2 weeks on B5 medium and then transferred to iron-sufficient or deficient medium for 3 days. Ferric-chelate reductase was determined as described previously [14]. Twenty seedlings were pooled and assayed as a group. Each value is the mean of five independent trials. The entire experiment was repeated four times. Statistical analysis of activity data was performed using a Student's $t$-test.

\section{Elemental analysis}

Wild-type (Ler) and 35S::DwMYB2 transgenic plants were grown for 14 days on B5 medium and then transferred to iron-sufficient or -deficient medium for 3 days. Root and shoot tissues were separately harvested, washed to exclude the contamination of $\mathrm{Fe}$ from medium and then dried overnight in a $65^{\circ} \mathrm{C}$ oven. Elemental analysis was performed using inductively coupled plasma-optic emission spectrometer (ICP-OES) at the Institute of Genetics and Developmental Biology, Chinese Academy of Sciences, as described [29]. Standard deviations were calculated for three biological replicates.

\section{Microarray analysis}

Root and shoot tissues were harvested from 14-day plantlets of wild-type (Ler) and $35 S: \because D w M Y B 2$ transgenic plants grown on normal B5 medium. Total RNA was reversely transcribed, labeled and hybridized to individual ATH1 Affymetrix chips at the State Key Laboratory of Plant Physiology and Biochemistry, China Agricultural University. Expression data was analyzed using GeneTraffic (Iobion Informatics, La Jolla, CA, USA). The determination of statistical significance was performed using the Cyber-T statistics program (http://visitor.ics. uci.edu/genex/cybert).

\section{Results}

Characterization of 35S::DwMYB2 transgenic Arabidopsis

The DwMYB2 driven by the constitutive $35 \mathrm{~S}$ promoter was cloned into pCAMBIA1300 binary vector and was introduced into Arabidopsis via Agrobacterium-mediated vacuum infiltration [28]. Fourteen hygromycin-resistant $\left(H y g^{R}\right)$ T1-independent transgenic lines harboring the $35 S:: D w M Y B 2$ transgene were obtained. Among them, 10 independent transgenic lines showed consistent phenotypes with variations in size and the morphology of rosette leaves in T2 seedlings grown on MS medium. The copy number of T-DNA insertion in these transgenic lines and their homozygosity were determined by Southern analysis and $H y g^{R}$ segregation (data not show). Two lines, DwMYB2-1
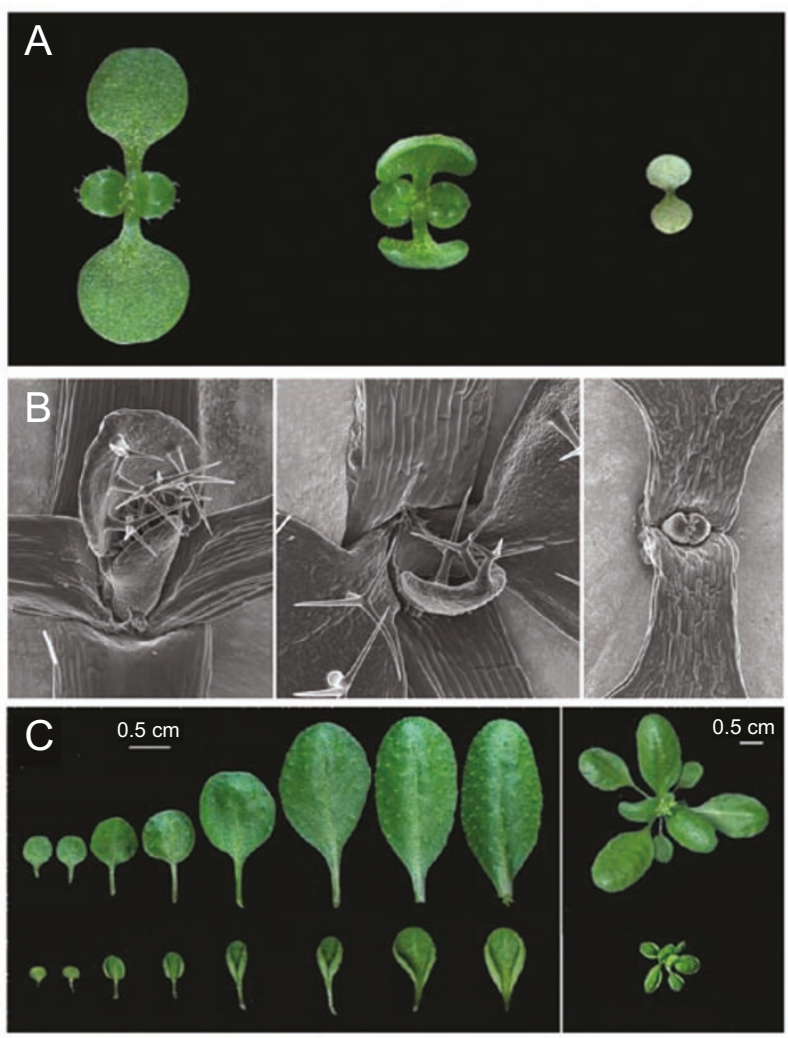

Figure 1 Phenotype of $35 S:: D w M Y B 2$ transgenic plants. (A) Nineday-old plants grown in soil. Left: wild type; middle: heterozygotes; and right: homozygotes. (B) SEM micrograph of plants shown in (A). (C) Comparison of cauline leaves (left) and seedlings (right) from 20-day-old wild-type (upper panel) and 35S::DwMYB2 heterozygous plants (lower panel) grown in soil (left panel). 
and DwMYB2-2, containing a single copy of the transgene were chosen for further study.

Three types of siblings were observed within either DwMYB2-1 or DwMYB2-2 segregating T2 progenies. Type I seedlings were phenotypically similar to wild type; in Type II seedlings, representing $35 S:: D w M Y B 2$ heterozygous plants, new leaves turned yellow and had curved cotyledon; while in Type III seedlings leaves were also yellowish and arrested at two-leaf primordial stage, and they died eventually, Type III seedlings were homozygous for the $35 S:$ : DwMYB2 transgene (Figure 1A). SEM showed that the first two leaf primordia were formed in both homozygous and heterozygous transgenic plants (Figure 1B). The leaf primordia were able to develop further in heterozygotes, although much slower compared to wild-type seedlings (Figure 1B). Both cotyledon and young leaf were pointed upright and curved inward (Figure 1A and 1C) irrespective of iron availability, indicating it was not related to iron deficiency. Leaves and seedlings were much smaller in $35 S:: D w M Y B 2$ homozygote compared to that of wild type (Figure 1C). To further clarify the seedling phenotype, T2 seeds were directly sowed to soil. The size reduction in seedlings and leaf phenotype were reproducible in soil. Most type III seedlings failed to grow up, and only a few

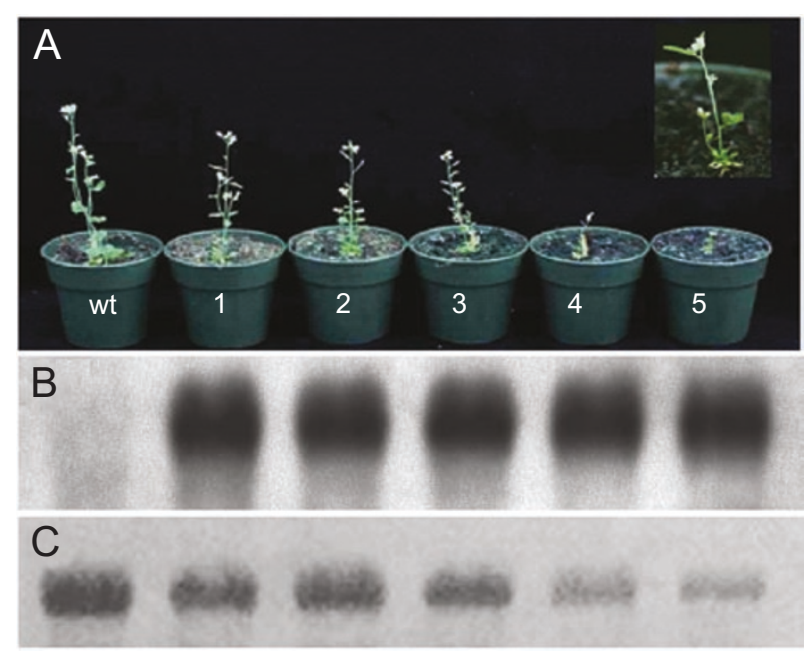

Figure 2 Northern analysis of $D w M Y B 2$ expression in T2 35S:: $D w M Y B 2$ transgenic plants. (A) Seedling phenotypes 40 days after germination. From left to right: wild type, 1, 2 and 3 are $35 S:$ : $D w M Y B 2$ heterozygotes; 4 and 5 are $35 S:: D w M Y B 2$ homozygotes. Inset: Magnification of plant in 5. (B) Northern blot hybridized with $D w M Y B 2$ cDNA probe. RNA was isolated from wild-type and T2 transgenic siblings shown in (A). Five micrograms or less RNA was loaded in each lane and hybridized with DIG-labeled probe. (C) The same blot as in (B) was hybridized with $28 \mathrm{~S}$ probe and used as internal control. Note that less RNA was loaded in lanes 5 and 6 than other lanes. could continue to grow and set a few flowers. The phenotype showed a typical 1:2:1 Mendelian segregation in the DwMYB2-1 and DwMYB2-2 T2 siblings, indicating that it is most likely linked to the transgene.

To further investigate whether the phenotype is a result of $D w M Y B 2$ overexpression, we performed Northern analysis on five transgenic individuals varying in severity of the phenotype (Figure 2A). Results showed that $D w M Y B 2$ mRNA levels in plants 4 and 5 was about 2-5 times higher compared to that in plants 1,2 and 3 (Figure 2B), indicating that the higher DwMYB2 expression level coincides with the severity of the phenotype. In fact, plants 1, 2 and 3 were heterozygous for the transgene, and plants 4 and 5 were homozygous as demonstrated by their Mendelian segregation of the $H y g^{R}$ gene in T3 generation and Southern analysis. No expression was detected in the wild-type plant (Figure 2B). Less RNA from homozygous lines was loaded as demonstrated by $28 \mathrm{~S}$ rRNA level (Figure $2 \mathrm{C}$ ), because the homozygous plants are very tiny and therefore rather difficult to obtain more RNA for analysis. Independent transgenic lines all showed similar phenotype and the severity of the phenotype correlated well with the $D w M Y B 2$ expression level, demonstrating that the level of $D$ WMYB2 expression in Arabidopsis caused the variation of the phenotype.

\section{DwMYB2 confers Arabidopsis hypersensitivity to iron deficiency}

Transgenic seedlings turned yellow after 9 days on MS medium and the whole seedling became yellow and some died within a month, whereas wild-type seedlings remained green. This yellow leaf phenotype in transgenic plants reminded us the typical symptom of iron deficiency that causes severe leaf chlorosis and seedling lethality in soil. To clarify the possibility of hypersensitivity to iron deficiency in DwMYB2-overexpressing plants, T2 seedlings containing wild-type, heterozygous, homozygous plantlets were first grown on the normal MS medium for 14 days (Figure 3A) and then transferred to fresh MS medium with iron (Figure 3B) or without iron (Figure 3C). After 3 days, the old yellow and newly formed leaves were all green on MS medium supplemented with iron (Figure 3B), whereas leaves of seedlings grew on MS medium without iron remained all yellow (Figure 3C). Similarly, 14-dayold yellow seedlings (Figure 3D) turned green if $10 \mathrm{ml}$ of $0.05 \mathrm{mM}$ FeNaEDTA was directly applied to the medium plate (Figure 3E). On the contrary, they remained yellow if water or other organic elements were added (Figure 3F). The homozygous $35 S:: D w M Y B 2$ can never fully recover, although they underwent limited growth under these conditions. These results demonstrated that $35 S:: D w M Y B 2$ transgenic plants can be recovered by transferring them to 

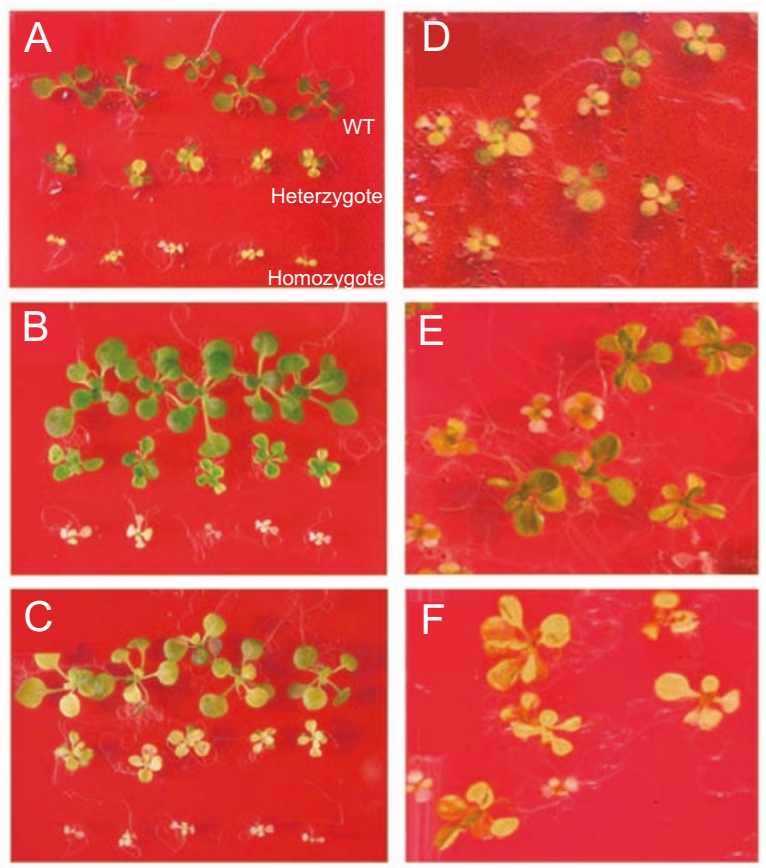

Figure 3 Iron deficiency phenotype in $35 S:: D w M Y B 2$ transgenic and wild-type seedlings. (A) Fourteen-day-old seedlings grew on normal MS medium before transfer to fresh medium with (B) or without (C) FeNaEDTA, respectively, in T3 generation. Upper panel: Wild type; middle panel: $35 S:: D w M Y B 2$ heterozygotes; and lower panel: $35 S:: D w M Y B 2$ homozygotes. (B) The seedlings from panel A grew on new medium with FeNaEDTA for 3 days. (C) The seedlings from panel A grew on new medium without FeNaEDTA for 3 days. Note that the new leaves are green in (B). (D) Fourteen-day-old seedlings of $\mathrm{T} 2$ heterozygotes progeny grew on MS medium with hygromycin. (E) Three days after FeNaEDTA addition. (F) Three days after $\mathrm{H}_{2} \mathrm{O}$ addition.

either fresh MS medium containing iron or direct application of iron, indicating that expression of $D w M Y B 2$ caused Arabidopsis plant hypersensitive to iron deficiency.

DwMYB2 enhances AtIRT1, AtIRT2 and AtFRO2 expression in roots under iron-deficient condition in Arabidopsis

Arabidopsis, belonging to the strategy I plants, responds to iron limitation by increasing its proton extrusion, which leads to acidification of the rhizosphere; Fe(III) is then reduced by membrane-associated ferric-chelate reductases and imported into the roots by Fe(II) transporters. Several genes that participate in these processes have been characterized very recently. Among them, ferric-chelate reductase (AtFRO2) is involved in iron uptake from soil [6]. The AtFRO2 gene expression is induced upon iron deficiency. AtIRT1 [16] and AtIRT2 [15] encode root periphery iron transporters and are also responsive to iron-deficient condition.
A
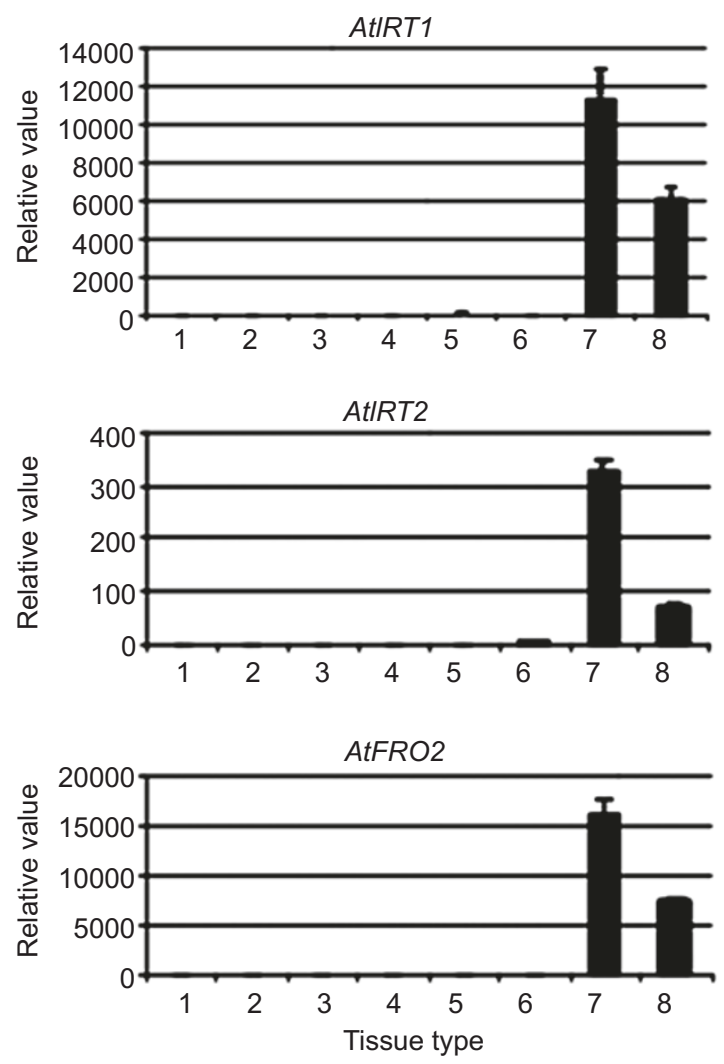

B

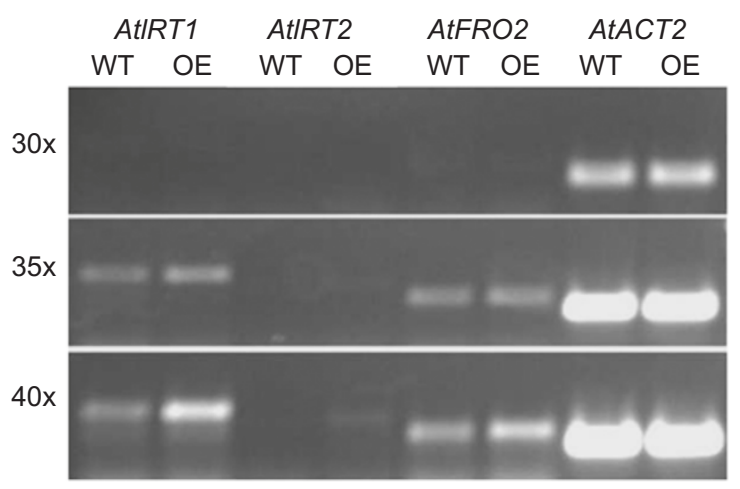

Figure 4 Iron-responding genes are up-regulated in the root of $35 \mathrm{~S}:$ : $D w M Y B 2$ transgenic lines. (A) Real-time PCR result of three genes, AtIRT1, AtFRO2 and AtIRT2, in different tissue under iron-deficient or iron-sufficient condition. 1, 35S::DwMYB2 transgenic line shoots under Fe-rich medium; 2, wild-type shoots under Fe-rich medium; 3, 35S::DwMYB2 transgenic line shoots under Fe-deficient medium; 4, wild-type shoots under Fe-deficient medium; 5, 35S::DwMYB2 transgenic line roots under $\mathrm{Fe}$-rich medium; 6, wild-type roots under Fe-rich medium; 7, 35S::DwMYB2 transgenic line roots under Fedeficient medium; and 8 , wild-type roots under Fe-deficient medium. (B) cDNA transcript of total RNA of wild-type, and 35S::DwMYB2 transgenic line is used as template and amplified with gene-specific primers by RT-PCR. AtIRT1, AtIRT2 and AtFRO2 genes were amplified for 30 cycles (upper panel), 35 cycles (middle panel) and 40 cycles (lower panel). The $A C T-2$ gene is used as internal control. 
To determine whether AtFRO2, AtIRT1 and AtIRT2 genes are up-regulated or not in $35 S: \because D w M Y B 2$ transgenic plants, we used real-time RT-PCR to monitor their expression. AtIRT1, AtIRT2 and AtFRO2 were only detected in wild-type roots grown under iron-deficient condition as reported previously (Figure 4A) $[6,16]$. No or very low level of AtIRT1, AtIRT2 and AtFRO2 expressions were detected in the shoot under either iron-rich or iron-deficient conditions (Figure 4A). In roots under iron deficiency, the expression level of AtIRT1 and AtFRO2 was two-fold higher in $35 S: \because D w M Y B 2$ transgenic plant compared to that in wild type; AtIRT2 expression level was about fourfold of the wild type grown under iron-deficient condition. The overall expression level of AtIRT1 and AtFRO2 was much higher than AtIRT2 in roots grown under iron deficiency (Figure 4A). We then detected the expression level of AtIRT1, AtIRT2 and AtFRO2 in roots of wildtype and $35 S: \because D w M Y B 2$ transgenic plants grown under iron deficiency by semi-quantitative RT-PCR, the result is consistent with the real-time PCR data (Figure 4B). We also detected $\mathrm{Fe}$ (III)-chelate reductase activity in wild-type and the transgenic plants roots grown under either iron-rich or iron-deficient conditions, AtFRO2 activity was about two-fold higher in $35 S: \because D w M Y B 2$ transgenic plant than in wild type under either iron-rich or iron-deficient conditions (Figure 5), consistent with its high expression level in transgenic plants. These data indicate that $D w M Y B 2$ enhances AtIRT1, AtIRT2 and AtFRO2 expression in irondeficient condition.

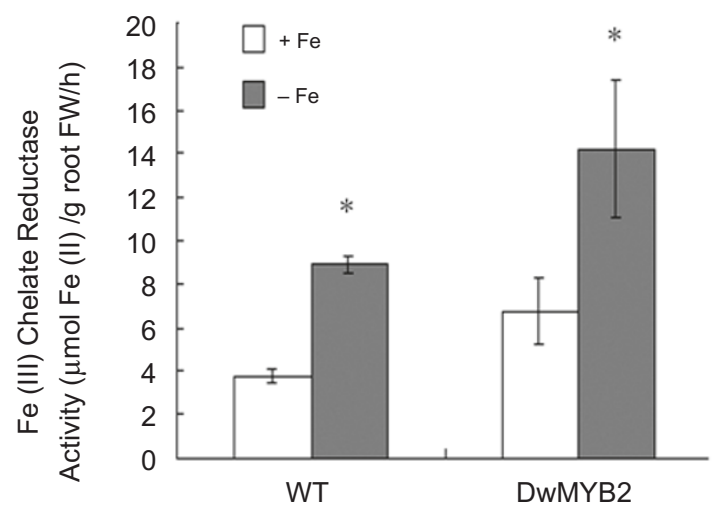

Figure 5 Assay of ferric-chelate reductase activity. Wild-type and $35 S:: D w M Y B 2$ transgenic lines were grown on standard B5 plates for 14 days and then transferred to either iron-deficient or -sufficient medium for 3 days. Fe(III)-chelate reductase activity of a pool of 20 plant roots was measured using the ferrozine assay [14]. Error bars indicate standard deviation of the five assays. * Significant differences at $P<0.05$.

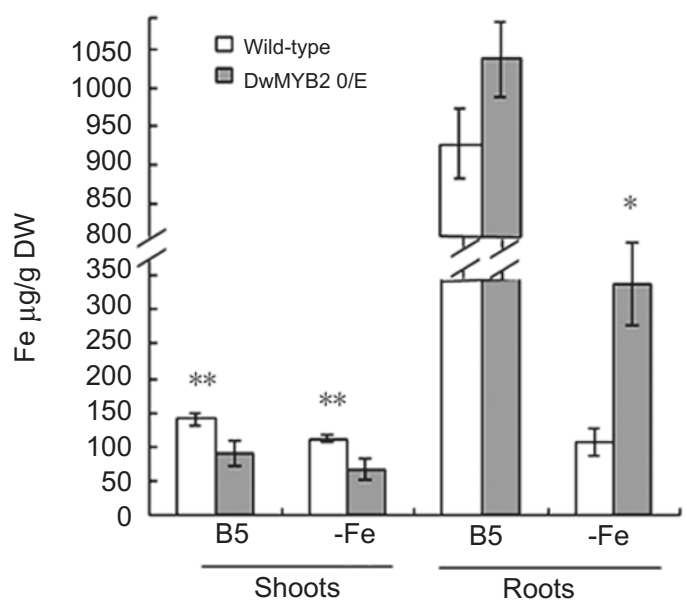

Figure 6 Imbalanced iron accumulation in $35 S:: D w M Y B 2$ transgenic line. Wild-type and $35 S:: D w M Y B 2$ transgenic lines were grown on B5 plates for 14 days, then transferred to iron-deficient (-Fe) or new B5 plates for 3 days. Plants were pooled and harvested into root and shoot samples, and three biological sets of tissue were subjected to elemental analysis. Standard deviations were calculated. * and **Significant differences at $P<0.05$ and $P<0.01$, respectively.

Root-to-shoot iron transportation was impaired in $35 S::$ DwMYB2 transgenic plants

To determine if $D w M Y B 2$ transgenic plants display altered iron accumulation, the iron content of $35 S:: D w$ MYB2 transgenic line and wild-type 17-day-old seedlings was measured by ICP-OES. The roots of $35 S: \because D w M Y B 2$ transgenic plants have three times more iron compared to that of wild-type plants when grown under iron-deficient conditions (Figure 6). However, there was no significant difference in root iron content between wild-type and transgenic plants grown on standard B5 medium, indicating that transgenic roots accumulate more iron compared to that of wild type under iron deficiency. On the contrary, shoots of transgenic plants accumulate less iron compared to that of wild type under either iron-rich or iron-deficient conditions. Compared to wild type, the shoots of $35 S:: D w-$ MYB2 transgenic plants contain about $40 \%$ less iron when grown under both conditions (Figure 6). Taken together, these data suggest that the iron transport from root to shoot is blocked in DwMYB2-expressing plants.

Genes involved in iron homeostasis were altered in $35 S::$ DwMYB2 transgenic plants

To further investigate what genes are regulated by the DwMYB2 transcription factor, DNA microarray experiments were carried out using Affymetrix ATH1 genechip. In total, 272 genes are down-regulated, and 520 genes are up-regulated with at least two-fold changes in expres- 
Table 1 Microarray analysis of 34 genes involved in iron homeostasis in Arabidopsis

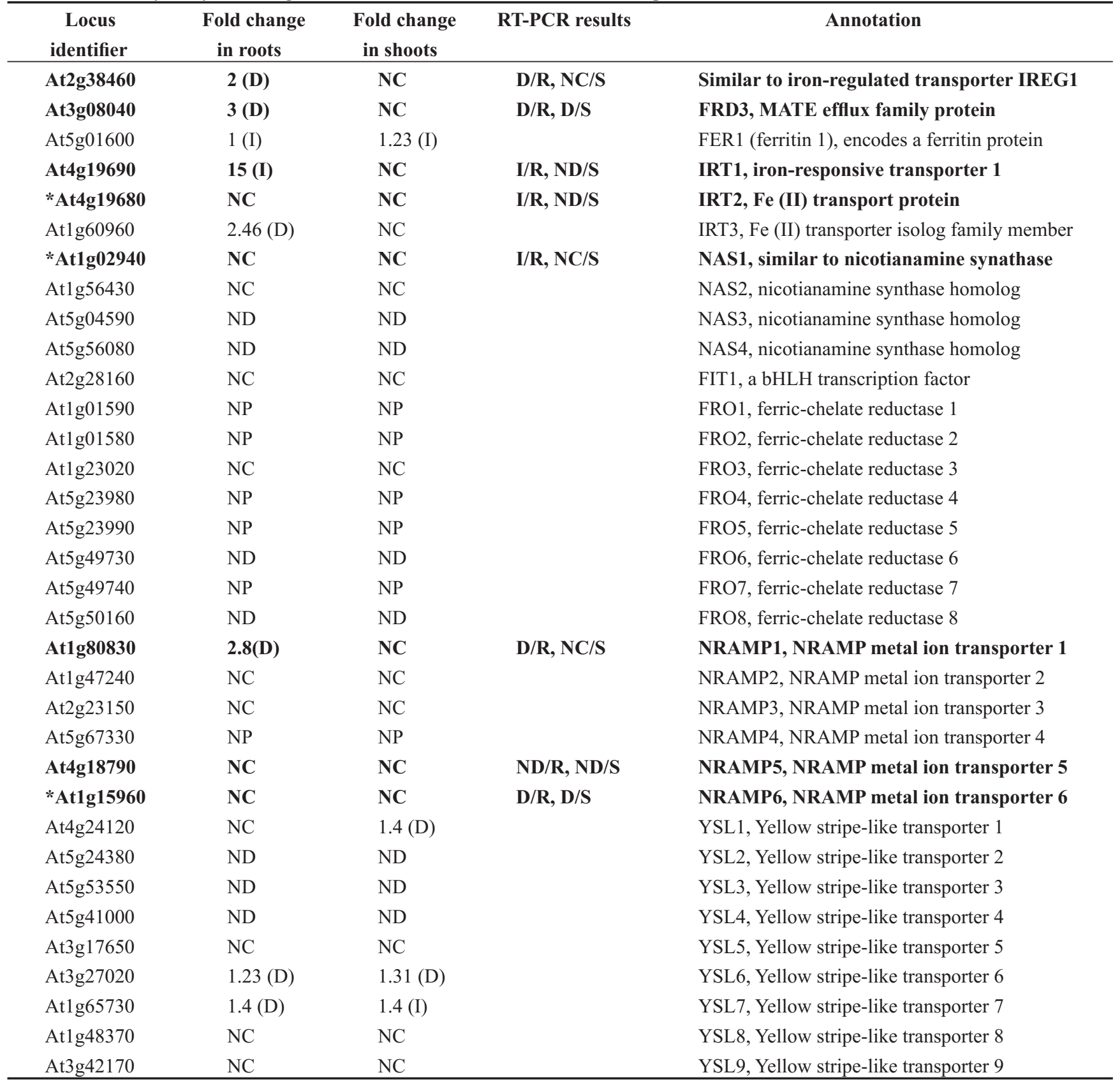

Expression fold changes of 34 genes known to be involved in iron transport or homeostasis were listed here. Fold change in root and shoot indicate the fold change between transgenic line shoot/root and wild-type shoot/root. I: induced; D: decreased; NC: no change; NP: not present on ATH1 genechip; ND: no detected; S: shoot; R: root; D/S: decreased in shoot. * indicating that although these genes showed no expression change by microarray because of their lower expression level, RT-PCR result showed different expression level. Items in bold showed RT-PCR-verified genes.

sion level in $35 S:: D w M Y B 2$ transgenic roots compared with wild-type roots, while in $35 S: \because D w M Y B 2$ transgenic shoots, 654 genes are down-regulated and 899 genes are up-regulated.

Among the 34 genes possibly involved in iron ho- meostasis, seven genes did not show detectable signal in our microarray analysis. In addition, six genes including AtFRO2 are not represented on the Affymetrix ATH1 genechip. Among the 21 genes with detectable signal on the Affymetrix ATH1 genechip, only AtIRT1 showed signifi- 
cant up-regulation in transgenic roots, whereas other genes like AtFRD3, AtIREG1 and AtNRAMP1 genes all showed decreased expression level, especially for the MATE efflux family protein AtFRD3 and the metal transporter NRAMP1 (Table 1). In addition to iron transporters listed above, several ATP-binding cassette (ABC) transporters also displayed expression changes in transgenic plants (see Supplement 2).

To validate the microarray data, semi-quantitative RTPCR analysis was performed on several genes (Figure 7). AtIRT1 was indeed expressed in transgenic roots under Fe-sufficient condition and 45 cycles RT-PCR detected the mRNA signal. In contrast, AtIREG1 was down-regulated only in transgenic roots. The MATE efflux family protein AtFRD3 was slightly down-regulated in roots and shoots of transgenic line. Transporter AtNRAMP1 was down-regulated only in transgenic roots. Some genes like AtIRT2, AtNAS1 and AtNRAMP6 with lower expression level showed no expression change in microarray analysis, but RT-PCR result showed different expression level in these genes. AtIRT2 was up-regulated in transgenic roots (Figure 4); AtNAS1 expression was only increased in shoot; and AtNRAMP6 was decreased both in shoots and roots. We did not detect the signal of AtNRAMP5 in either shoots or roots. Among the two ABC transporters (At5g60740 and At1g15520), At5g60740 was not detectable in either shoots or roots, and At 1 g15520 was down-regulated only in the shoot (Figure 7; Table 1). These data indicated that

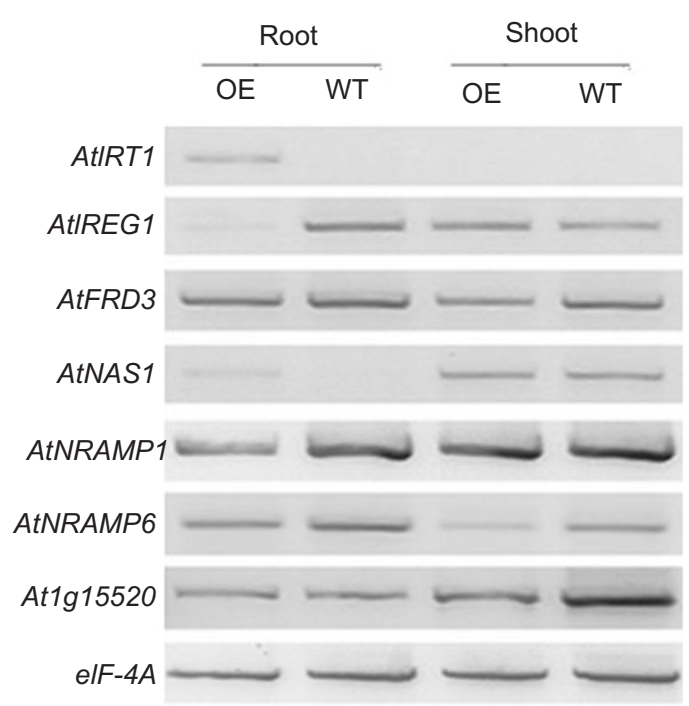

Figure 7 Expression level of iron-related genes in wild-type and $35 S:$ : $D w M Y B 2$ transgenic lines. The gene transcript levels in the shoot and root of wild-type and $35 S:: D w M Y B 2$ transgenic line were analyzed by RT-PCR analysis using gene-specific oligonucleotides described in Supplement 1. eIF-4A gene was used as loading control.
DwMYB2 transcription factor indeed altered the expression of many genes involved in iron homeostasis in transgenic Arabidopsis.

\section{Discussion}

Iron uptake, subsequent translocation from roots to shoots and intracellular allocation are complicated and tightly regulated processes. Although great progress has been made in understanding the molecular mechanism of iron uptake in the last decade, little is known about iron translocation in plants. The data presented here demonstrated that the expression of the orchid R2R3-MYB gene DwMYB2 in Arabidopsis promoted iron uptake and accumulation in roots and impaired the iron transportation from roots to shoots, converting the transgenic plants hypersensitive to iron limitation. The plants exhibited chlorotic leaves, a typical symptom of iron deficiency. Application of iron to the medium or transferring the yellow seedlings to fresh MS medium partially rescued this symptom. Additionally, lower iron content was present in shoots of $35 S:: D w M Y B 2$ transgenic line compared to that of the wild type, even though the iron content in their roots was higher than the wild type. One likely explanation is that the expression of DwMYB2 in $35 S:: D w M Y B 2$ transgenic seedlings alters the expression level of some key genes involved in iron translocation or metabolism, resulting in the disruption of the iron distribution and homeostasis in the plants. Consequently, the transgenic plants were more sensitive to iron deficiency and required higher iron concentration for their growth.

The ferric-chelate reductase AtFRO2 and the ferrous uptake transporters AtIRT1 and AtIRT2 are three wellcharacterized genes responsible for responding to iron deficiency in roots of the model plant Arabidopsis [5-7, $15,16,30-32]$. They are regulated by signals generated in shoots or in roots based on the iron status $[17,33]$. The ectopic expression of the DwMYB2 gene in Arabidopsis enhanced the expression of the three iron uptake response genes even though the roots of $35 S:: D w M Y B 2$ transgenic line contained higher iron content compared to that of the wild type both under iron deficiency or normal B5 medium conditions (Figure 6). Considering that the iron content is higher in roots and lower in shoots of the transgenic plants in comparison with the wild type, we deduced that the enhanced transcription of AtFRO2, AtIRT1 and AtIRT2 in $35 S:: D w M Y B 2$ transgenic line might be controlled by a shoot-derived iron deficiency signal, and that the higher iron accumulation in roots of the $35 S: \because D w M Y B 2$ transgenic line might be the consequence of the enhanced expression of the three genes.

As described above, expressing the DwMYB2 in Arabi- 
dopsis led to an imbalance of iron distribution in roots and shoots of the transgenic plants. Significantly less iron was transported from roots to shoots in the plants under either iron-deficient or normal B5 medium conditions, although their roots contained more iron compared with the wild type, indicating that the iron transport from roots to shoots was impaired in the transgenic plants. Iron, once taken up by the root, must be loaded from the root cortical cells to the xylem, then transported through the pipeline of xylem to shoots and finally allocated to leaf cells for assimilation. The influx and efflux of iron in xylem are likely controlled by different transporters. Considering the fact that the $35 S:$ : $D w M Y B 2$ transgenic line contains lower iron content in shoots and a higher content in roots compared with the wild type, we suggest that the impairment of the iron translocation in the transgenic plants might happen in the process of xylem influx in roots rather than in efflux in shoots. Some genes involved in the processes of iron short- or long-range transports have been reported. AtFRD3, a MATE efflux family protein, putatively functions in root xylem loading of an iron chelator or other factor necessary for efficient iron uptake out of the xylem or apoplastic space and into leaf cells of Arabidopsis [34]. The frd3 mutant showed a similar phenotype as the $35 S:: D w M Y B 2$ transgenic line, such as chlorosis and enhanced expression of the iron deficiency response genes AtFRO2 and AtIRT1 under iron-sufficient condition, with an exception that the $\mathrm{Fr} d 3$ mutant accumulated iron in shoots, while the $35 S: \because D w M Y B 2$ transgenic line accumulated iron in roots [21]. Initial microarray analysis showed that the transcription level of AtFRD3 was decreased about three-fold in roots of the transgenic plants. However, it was not clearly confirmed by subsequent RT-PCR analysis. According to different iron accumulation pattern, it is unlikely that the impaired iron allocation in the $35 S:: D w M Y B 2$ transgenic line is a consequence of the weakly reduced $A t F R D 3$ transcription.

NA, a polyamine, is a well-known metal chelator involved in iron allocation in plants [35]. Same as $\mathrm{frd} 3$ mutant, the NA-free mutant chloronevera ( $c h l n)$ of tomato displays iron-deficient chlorosis in shoots, constitutive expression of the iron deficiency response genes LeFRO1 and LeIRT1 in roots and accumulates more iron in its leaves than the wild type [9, 11, 36, 37]. In the genome of Arabidopsis, there are four genes termed AtNAS1 to AtNAS4 responsible for NA synthesis. Real-time PCR analysis showed that no significant alteration in the transcript abundance of the four genes was detected in the transgenic plants compared to the wild type (data not shown).

Additionally, NRAMP and YSL families of metal transporters are also involved in iron allocation in plants [38]. Our microarray and RT-PCR analysis results indicated that all NRAMP and YSL genes of the Arabidopsis did not show severe changes in their expression level, except for AtNRAMP1 and AtNRAMP6. AtNRAMP1 was involved in iron transport in $A$. thaliana [19]. Its transcript accumulated under iron deficiency in roots and overexpression of $A t N-$ RAMP1 in Arabidopsis led to increase in plant resistance to toxic iron concentration, suggesting that the AtNRAMP1 protein might be involved in intracellular iron transport to sequestering compartments for iron detoxification in plants. Therefore, it is also unlikely that some reduced transcript of AtNRAMP1 in roots of the $35 S:: D w M Y B 2$ transgenic line is the main reason for the iron distribution disruption in the transgenic plants. The precise functions of AtNRAMP6 remain unknown and the expression of AtNRAMP6 was decreased both in roots and shoots of $35 S:: D w M Y B 2$ transgenic line, so we could not exclude the possibility of a potential role of AtNRAMP6 in iron transportation from root to shoot.

IREG1 gene encodes an iron-regulated transporter involved in the basolateral efflux of iron from epithelial cells to the circulation [22]. The homolog of the IREG1 in Arabidopsis genome is At2g38460, here after termed AtIREG1. The biological functions of AtIREG1 are still unclear. Curie and Briat [38] assumed that AtIREG1 might encode an iron transporter and be involved in the vessel loading of iron. Our microarray data indicate that the transcript level of AtIREG1 was decreased two-fold in the roots of $35 S: \because D w M Y B 2$ transgenic line in comparison with the wild type. It was well confirmed subsequently by RT-PCR analysis. These results suggest that the deceased transcription level of AtIREG1 in the DwMYB2 transgenic plants might be a possible reason for the disruption of iron translocation in the $35 S: \because D w M Y B 2$ transgenic line. Further characterization of the knockout mutant of AtIREG1 will be helpful to clarify it.

Besides genes described above, $\mathrm{ABC}$ transporters are also possibly involved in iron transportation and allocation in higher plants. There are 103 potential functional ABC transporters in the Arabidopsis genome, but only a few have been postulated to be responsible for transport of inorganic ions or organic ions into isolated vacuole or tonoplast vesicles [39]. The microarray analysis data revealed that there were $11 \mathrm{ABC}$ transporters presenting a changed expression level in roots and shoots of the transgenic plants compared with the wild type (see Supplement 2). Six of them displayed an increased expression level and two displayed decreased expression in shoots; in roots two genes showed a decreased expression. Therefore, the four genes showing a reduced expression level in the $35 S:: D w M Y B 2$ transgenic line are also possible candidate genes involved in iron transportation. Further analysis of their mutants will give a clarification. Other transporters, including peptide, phosphate, nitrate and sugar transporters, also repressed 


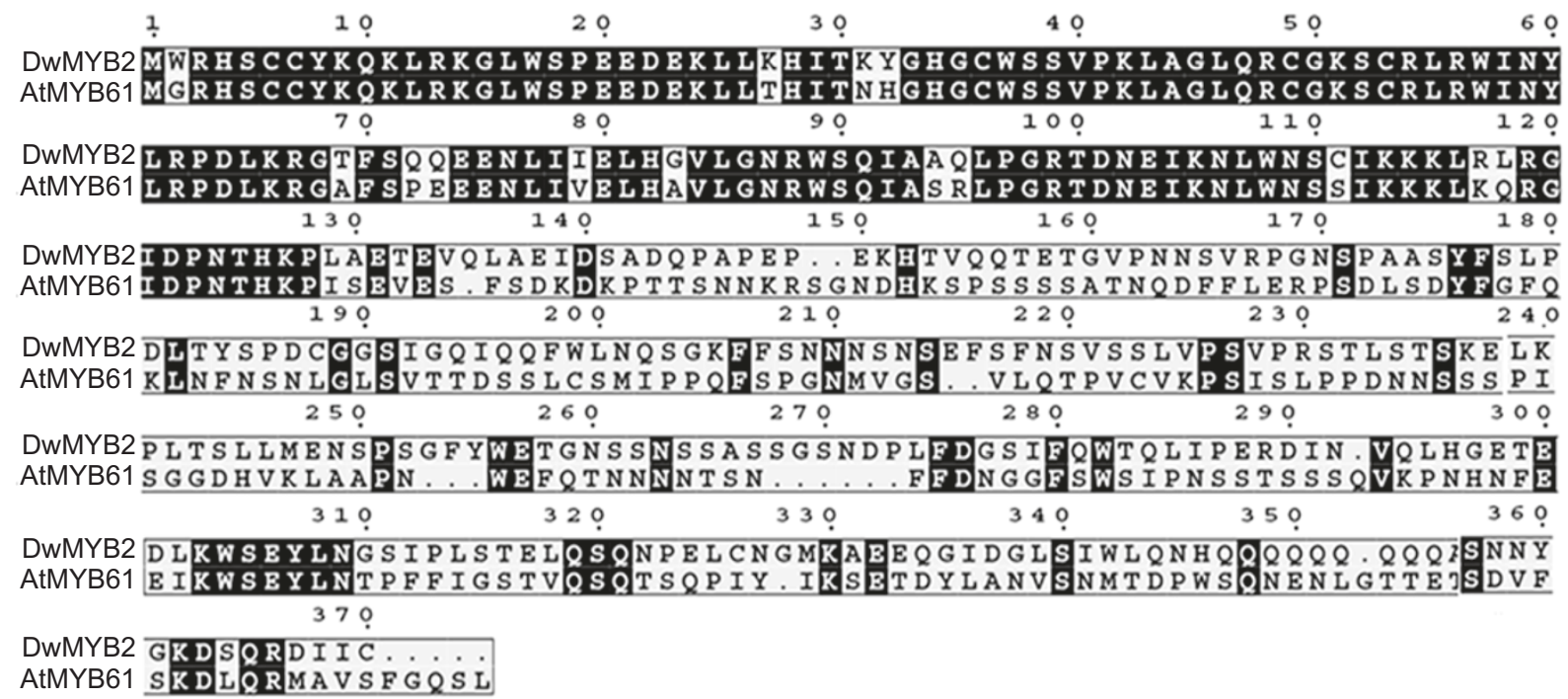

Figure 8 Alignment between DwMYB2 and its homolog AtMYB61. Two proteins share 42\% identity in full-length amino acid sequence, $95 \%$ identity in R2R3 domain and $18 \%$ identity in C-terminus.

or induced in transgenic plants (see Supplement 2). These might be due to the secondary effects of the imbalanced iron distribution.

DwMYB2 is a heterologous gene for the Arabidopsis genome. The closest homolog in Arabidopsis is AtMYB61, which shares $42 \%$ overall identity at amino acid level with DwMYB2. The homologous region mainly lies in the R2R3 domain (95\% identity), with little homology in C-terminus region (18\% identity) (Figure 8). AtMYB61 knockout mutants were deficient in seed mucilage extrusion upon imbibitions [40], and AtMYB61-overexpressing transgenic plants showed ecotopic lignification and dark photomorphogenic phenotype [41]. These data indicate that AtMYB61 and DwMYB2 are not functional homologs and may have different functions in plant development, despite the fact that they share a conserved DNA-binding $\mathrm{R} 2 \mathrm{R} 3$ domain. It is known that functional diversion of the $M Y B$ gene mainly depends on the structure of the C-terminus [25]. Therefore, it would be interesting to know the expression pattern of DwMYB2 in orchid, for example, by determining whether its expression is expressed in the root vasculature or it is responsive to iron deficiency. This will provide clues about its native function in iron homeostasis in orchid.

In conclusion, expression of the ectopic $D w M Y B 2$ gene in $A$. thaliana clearly affected iron allocation and homeostasis in the transgenic plants, conferring Arabidopsis plants hypersensitive to iron limitation. Microarray analysis showed that more than 1000 genes in $35 S:: D w M Y B 2$ transgenic line altered their transcript level compared with the wild type. Since the addition of iron restores the chlorotic phenotype, most likely that the majority of the expression changes are secondary effects of the disrupted iron translocation and homeostasis. The decreased transcription level of AtIRE G1, AtNRAMP6 and the four ABC transporters might be the possible reason(s) for the impaired iron allocation in the DwMYB2-expressing Arabidopsis plants. Further characterization will give new insight for understanding of iron allocation in plant.

\section{Acknowledgments}

This work was supported by " 100 Talents Programme of the Chinese Academy of Sciences" to Yang WC, Chinese Academy of Sciences, China, by a Distinguished Young Scholars grant of the Chinese National Science Foundation (No. 30225029) and by the Chinese Ministry of Science and Technology (No. 2005cb20904) to Ling HQ.

\section{References}

1 Guerinot ML, Yi Y. Iron: nutritious, noxious and not readily available. Plant Physiol 1994; 104:815-820.

2 Helliwell B, Gutteridge JMC. Biologically relevant metal ion-dependent hydroxyl radical generation. FEBS Lett 1992; 307:108-112.

3 Marschner H. Mineral Nutrition of Higher Plants, 2nd Edition. San Diego: Academic Press, 1995.

4 Colangelo EP, Guerinot ML. The essential basic helix-loop-helix protein FIT1 is required for the iron deficiency response. Plant Cell 2004; 16:3400-3412. 
5 Eide D, Broderius M, Fett J, Guerinot ML. A novel iron-regulated metal transporter from plants identified by functional expression in yeast. Proc Natl Acad Sci U S A 1996; 93:5624-5628.

6 Robinson NJ, Procter CM, Connolly EL, Guerinot ML. A ferric-chelate reductase for iron uptake from soils. Nature 1999; 397:694-697.

7 Vert G, Grotz N, Dedaldechamp F, et al. IRT1, an Arabidopsis transporter essential for iron uptake from the soil and for plant growth. Plant Cell 2002; 14:1223-1233.

8 Eckhardt U, Mas Marques A, Buckhout TJ. Two iron-regulated cation transporters from tomato complement metal uptake-deficient yeast mutants. Plant Mol Biol 2001; 45:437-48.

9 Ling H-Q, Koch G, Baeumlein H, Ganal MW. Map-based cloning of chloronerva - a gene involved in iron uptake of higher plants encoding nicotianamine synthase. Proc Natl Acad Sci U S A 1999; 96:7098-7103.

10 Ling H-Q, Bauer P, Bereczky Z, Keller B, Ganal M. The tomato FER gene encoding a bHLH protein controls iron-uptake responses in roots. Proc Natl Acad Sci U S A 2002; 99:1393813943.

11 Li L, Cheng X, Ling HQ. Isolation and characterization of Fe(III)chelate reductase gene LeFRO1 in tomato. Plant Mol Biol 2004; 54:125-136.

12 Waters BM, Blevins DG, Eide DJ. Characterization of FRO1, a pea ferric-chelate reductase involved in root iron acquisition. Plant Physiol 2002; 129:85-94.

13 Fox TM, Guerinot ML. Molecular biology of cation transport in plants. Annu Rev Plant Physiol Plant Mol Biol 1998; 49:669696.

14 Yi Y, Guerinot ML. Genetic evidence that induction of root $\mathrm{Fe}$ (III) chelate reductase activity is necessary for iron uptake under iron deficiency. Plant J 1996; 10:835-844.

15 Vert G, Briat JF, Curie C. Arabidopsis IRT2 gene encodes a root-periphery iron transporter. Plant J 2001; 26:181-189.

16 Connolly EL, Fett JP, Guerinot ML.. Expression of the IRT1 metal transporter is controlled by metals at the levels of transcript and protein accumulation. Plant Cell 2002; 14:1347-1357.

17 Vert GA, Briat JF, Curie C. Dual regulation of the Arabidopsis high-affinity root iron uptake system by local and long-distance signals. Plant Physiol 2003; 132:796-804.

18 VonWirén N, Klair S, Bansal S, et al. Nicotianamine chelates both FeIII and FeII. Implications for metal transport in plants. Plant Physiol 1999; 119:1107-1114.

19 Curie C, Alonso JM, Le Jean M, Ecker JR, Briat JF. Involvement of NRAMP1 from Arabidopsis thaliana in iron transport. Biochem J 2000; 347:749-755.

20 Thomine S, Wang R, Ward JM, Crawford NM, Schroeder JI. Cadmium and iron transport by members of a plant metal transporter family in Arabidopsis with homology to NRAMP genes. Proc Natl Acad Sci U S A 2000; 97:4991-4996.

21 Rogers EE, Guerinot ML. FRD3, a member of the multidrug and toxin efflux family, controls iron deficiency responses in Arabidopsis. Plant Cell 2002; 14:1787-1799.

22 McKie AT, Marciani P, Rolfs A, et al. A novel duodenal iron-regulated transporter, IREG1, implicated in the basolateral transfer of iron to the circulation. Mol Cell 2000; 5:299-309.

23 Curie C, Panaviene Z, Loulergue C, Dellaporta SL, Briat JF, Walker EL. Maize yellow stripel encodes a membrane protein directly involved in Fe(III) uptake. Nature 2001; 409:346-349.

24 Yuan YX, Zhang J, Wang DW, Ling H-Q. AtbHLH29 of Arabidopsis thaliana is a functional ortholog of tomato FER involved in controlling iron acquisition in strategy I plants. Cell Res 2005; 15:613-621.

25 Jin H, Martin C. Multifunctionality and diversity within the plant MYB-gene family. Plant Mol Biol 1999; 41:577-585.

26 Stracke R, Werber M, Weisshaar B. The R2R3-MYB gene family in Arabidopsis. Curr Opin Plant Biol 2001; 4:447-456.

$27 \mathrm{Wu}$ XM, Lim SH, Yang WC. Characterization, expression and phylogenetic study of $\underline{R} 2 R 3-M Y B$ genes in orchid. Plant Mol Biol 2003; 51:959-972.

28 Bechtold N, Ellis J, Pelletier G. In Planta agrobacterium-mediated gene transfer by infiltration of adult Arabidopsis thaliana plants. CR Acad Sci Paris Life Sci 1993; 316:1194-1199.

29 Lahner B, Gong J, Mahmoudian M, et al. Genomic scale profiling of nutrient and trace elements in Arabidopsis thaliana. Nat Biotechnol 2003; 21:1215-1221.

30 Connolly EL, Campell NH, Grotz N, Prichard CL, Guerinot ML. Overexpression of the FRO2 ferric chelate reductase confers tolerance to growth on low iron and uncovers posttranscriptional control. Plant Physiol 2003; 133:1102-1110.

31 Henriques R, Jasik J, Klein M, et al. Knock-out of Arabidopsis metal transporter gene IRT1 results in iron deficiency accompanied by cell differentiation defects. Plant Mol Biol 2002; 50:587-597.

32 Varotto C, Maiwald D, Pesaresi P, Jahns P, Salamini F, Leister D. The metal ion transporter IRT1 is necessary for iron homeostasis and efficient photosynthesis in Arabidopsis thaliana. Plant J 2002; 31:589-599.

33 Grusak MA, Pezeshgi S. Shoot-to-root signal transmission regulates root $\mathrm{Fe}(\mathrm{III})$ reductase activity in the dgl mutant of pea. Plant Physiol 1996; 110:329-334.

34 Green LS, Rogers EE. FRD3 controls iron localization in Arabidopsis. Plant Physiol 2004; 136:2523-2531.

35 Hell R, Stephan UW. Iron uptake, transport and stabilization in plants. Planta 2003; 216:541-551.

36 Stephan UW, Scholz G. Nicotianamine: mediator of transport of iron and heavy metals in the phloem? Physiol Plantarum 1993; 88:522-529.

37 Ling H-Q, Pich A, Scholz G, Ganal MW. Genetic analysis of two tomato mutants affected in the regulation of iron metabolism. Mol Gen Genet 1996; 252:87-92.

38 Curie C, Briat JF. Iron transport and signaling in plant. Annu Rev Plant Biol 2003; 54:183-206.

39 Yamaguchi H, Nishizawa NK, Nakanishi H, Mori S. IDI7, a new iron-regulated $\mathrm{ABC}$ transporter from barley roots, localizes to the tonoplast. J Exp Bot 2002; 53:727-735.

40 Penfield S, Meissner RC, Shoue DA, Carpita NC, Bevan MW. MYB61 is required for mucilage deposition and extrusion in the Arabidopsis seed coat. Plant Cell 2001; 13:2777-2791.

41 Newman LJ, Perazza DE, Juda L, Campbell MM. Involvement of the R2R3-MYB, AtMYB61, in the ectopic lignification and dark-photomorphogenic components of the $\operatorname{det} 3$ mutant phenotype. Plant J 2004; 37:239-250.

42 Sambrook J, Fritsch EF, Maniatis T. Molecular Cloning: A Laboratory Manual. 2nd Edition. Plainview, NY: Cold Spring Harbor Laboratory Press, 1989. 